\title{
Efficiency of Akkermansia muciniphila in type 2 diabetes and obesity
}

\author{
Farzaneh Mohammadzadeh Rostami ${ }^{1}$, Saman Shalibeik ${ }^{2}$, Bahram Nasr Esfahani ${ }^{1,}{ }^{*}$ \\ ${ }^{1}$ Department of Bacteriology and Virology, Faculty of Medicine, Isfahan University of Medical Sciences, Isfahan, Iran \\ 2 Department of Microbiology, Faculty of Biological Sciences, Falavarjan Branch, Islamic Azad University, Isfahan, Iran
}

\begin{abstract}
Akkermansia muciniphila is an anaerobic species of gut microbiome that has been proposed as a new functional microbiota with probiotic properties. Recent research has shown the amazing abilities of probiotic bacteria, A. muciniphila, which resides in most people's intestines. These bacteria affect the body if it increases or decreases abdominal fat. The presence of $A$. muciniphila has opened new ways for the use of this plentiful intestinal symbiont in next generation therapeutic products, as well as targeting microbiota dynamics. A. muciniphila is particularly effective in increasing mucosal thickness and enhancing bowel barrier function. As a result, host metabolic markers improve. The host functions that are disrupted in various diseases with a particular focus on metabolic disorders in animals and humans. A specific protein in the outer membrane of $A$. muciniphila called Amuc-110 could in the future be a strong candidate for drug production. As a result, we suggest that microbes and our microbiology or gut microbiome knowledge could be a new source for future treatments. The objectives of this review are to summarize the data available on the distribution of A. muciniphila gut in health and disease, to provide insights into the environment and its role in the creation of microbial networks at the mucosal interface, as well as to discuss recent research on its role in regulation.
\end{abstract}

Keywords: Akkermansia muciniphila; Type 2 diabetes; Obesity; Prebiotics

\section{Introduction}

Akkermansia was introduced by Dutch microbiologist Anton Akkermansia (born October 28, 1940: died August 21, 2006) who studies the biology of microorganisms. This genus contains a single species called Akkermansia muciniphila [1]. The word mucio is derived from the Latin root of mucin in the gastrointestinal tract and phila is derived from the Latin word filos. Muciniphila also calls mucin an inhibitor. A. muciniphila is a dominant human intestinal mucin-degrading bacterium in a genus

\footnotetext{
* Corresponding author:

Dr. Bahram Nasr Esfahani, Ph.D

Department of Bacteriology and Virology, School of Medicine, Isfahan University of Medical Sciences, Isfahan, Iran

Tel/Fax: +98 3137922478

Email: nasr@hlth.mui.ac.ir

https://orcid.org/oooo-0003-3264-3361
}

Received: June, 28, 2020

Accepted: August, 09, 2020 proposed in 2004 by Muriel Derrien and colleagues. It's a Gram-negative, oval-shaped, strictly anaerobic, non-motile non-spore-former. Odd that such an abundant organism had to wait so long to be discovered. Probably this has to do with the conditions used to its isolation, which is an anaerobic medium containing gastric mucin as the only carbon and nitrogen source. The genus Akkermansia belongs to the division Verrucomicrobia [2,3].

Microbial ecosystem of $A$. muciniphila Most likely, there is a relationship between fecal 
microbiota and health indicators. Not just a pure microorganism but an ecosystem that restores the complex balance between host biology and the environment. According to the abundant study of $A$. muciniphila, it is related to extensive metagenomics frequency and abundance of bacterial genes [4].

\section{Role of A. muciniphila in obesity and type 2 diabetes}

Obesity-related metabolic disorders and cardiac metabolism are widespread worldwide. Among all environmental factors, the intestinal microbiota is now an important intervention in host energy and metabolism that is susceptible to several noncommunicable diseases [5]. Among the nextgeneration bacteria that appear to be useful, $A$. muciniphila is the future candidate. Without a doubt, A. muciniphila is inversely related to obesity, diabetes, cardiovascular disease and superficial inflammation [2]. Gut microbiota and its changes in the composition and biodiversity, can play an appropriate role in the expansion of metabolic diseases. In humans, most of the papers show that a depauperated microbial diversity is associated with a higher insulin resistance, obesity and inflammation Although oral administration of $A$. muciniphila to mice fed with high-fat diet (HFD) extremely improves glucose homeostasis, the antidiabetic properties of this bacterium have not been indicated in humans because of its growth properties and oxygen sensitivity that set the use of living A. muciniphila improper for putative therapeutic chances. [6]. In addition, numerous documents have shown that pasteurization of $A$. muciniphila increases both stability and efficiency of strains [7]. This gives you the powerful A. muciniphila as the next generation of candidates to produce new foods or medicinal supplements with beneficial properties. Finally, a specific protein in the outer membrane of A. muciniphila, called Amuc_1100, which is one of the most abundant proteins involved in pellet formation, can be a powerful candidate for the production of subsequent drugs [8-11]. We suggest that microbes and microbiology, or our gut microbiome knowledge, could be a new source for future treatments. Obesity provides the basis for developing type 2 diabetes and cardiovascular disease. These two pathologies are part of the metabolic syndrome. It is also an important issue in public health [7]. Intestinal microbes play a vital part in regulating host metabolism and surface inflammation. Several researches have shown that our eating lifestyles greatly impact the composition and function of the gut microbiota and may ultimately play a role in initiating or protecting against metabolic disorders [12,13].

\section{From peribiotics to next-generation} beneficial microbes: focus on identifying $A$. muciniphila

One possible way to influence the intestinal microbiota, which is well documented, is to use selected microbes that market the host health benefits to the host by defining "living microorganisms that are used properly." Other methods, such as the use of probiotics over the past 20 years, have received much attention [14]. The concept of prebiotics, first observed by Gibson and Rutherford (1995), has led to a large number of dietary supplements, leading to significant market growth [15]. Peribiotics are the selective stimulation of the growth or activity of one or a limited number of microbial genera (species) or species in the gut microbiota that provide health benefits to the host [16]. According to the methodological and fundamental research of microbiologists, there has been a great deal of progress recently in our understanding of gut microbiota. However, the subject of peribiotics has not fully understood. Despite various research on the molecular mechanisms underlying how prebiotics and intestinal microbes interact with the host, the identification of the candidate bacterium elaborate in useful effects on energy, glucose, lipid metabolism and immunity is still difficult [17]. A. muciniphila is one of the most abundant single species in the human gut microbiota $(0.5 \%-0.5 \%$ of total bacteria) and in 2004 by Maurice Drine in his doctoral research at the University of Wageningen as a specialist in the use of the distinct and Specific Mucus $[18,19]$ The discovery began with the fact that the human body produces "Peribiotics" or microbial layers called mucus (sputum), which is a glycoprotein and offspring especially in the large intestine that destroy it goes [20]. While experiments on non-microbial mice have shown that $A$. muciniphila shows immunity and metabolic signaling, especially in the colon, the exact function of these microbes is unclear [21, 22]. Further evidence of the function of $A$. muciniphila has been repeatedly identified by other prebiotic studies using inulin-type fructans, initially known as bifidogenic compounds that can increase the quantity of Bifidobacterium species. They thanked the development of new techniques independent of 
culture. The study investigated the effect of this type of prebiotics on a microbial complex in mice [23]. The first surprise was that more than 100 different species were affected by prebiotics [14]. Among these bacteria, they found that the relative abundance of $A$. muciniphila increased more than 100-fold after peribiotic intake and reached more than $4.5 \%$ in highfat diets, whereas this effect on a normal diet $(2.5 \%$ 0.09) was less in his model [22]. Notably, these findings are approved in different sets of studies [23, 24]. Some studies show that $A$. muciniphila was lower in the gut microbiota of diabetic rats and mice that were genetically or affected by obese diets. However, few studies have reported the frequency of $A$. muciniphila in mice with increased glucose and fat intake [15]. Insulin-rich fructans have also been widely shown to show obesity-related metabolic disorders, including fat mass depletion, insulin resistance, hepatic steatosis, and intestinal barrier strengthening [25]. Importantly, in humans, the frequency of $A$. muciniphila has been reduced in several pathological conditions, including obesity, type 2 diabetes, gastrointestinal inflammation, hypertension, and intestinal disease [26, 27]. On the other hand, it has been found that antidiabetic therapies, such as the use of metformin and bariatric surgery, are associated with a significant increase in mucosal levels. Thus, much evidence has been shown that $A$. muciniphila can protect against specific metabolic disorders and risk factors for cardiovascular metabolism associated with a low degree of inflammation [28].

The role of A. muciniphila on and around the intestine

The gut microbiota is the largest human microbial reservoir that has a prominent role in human health and is involved in regulating many host physiological pathways such as regulating gastrointestinal stimulation, digestive barrier permeability, lipid distribution, and energy homeostasis [5]. A great deal of data suggesting the relative frequency of $A$. muciniphila in obesity and metabolic disorders in mice and humans, we decided to investigate the usual association between A. muciniphila and metabolic enhancers $[2,5,29]$. Carlota Dao et al found that the use of $A$. muciniphila, a live fillet with 2.108 bacterial cells per day, partially protected diet-induced obesity in mice [2]. Certainly, when dietary intake was not altered or dietary fat was removed, rats had a 50\% lower weight gain treated with the live drug $A$. muciniphila. This protection is reflected by less subcutaneous and internal fat mass as well as by increased markers of fatty acid oxidation (Acox1, Cpt1a, Acacb) in adipose tissue [30, 31]. In addition, animals treated with the live $A$. muciniphila substrate no longer showed insulin resistance, infiltrating inflammatory cells (CD11c) in adipose tissue which is one of the most important features of obesityassociated with superficial inflammation [32]. Interestingly, the metabolic improvements following the administration of live A. muciniphila were comparable with results obtained from the treatment with oligofructose or inulin [30,32]. However, live $A$. muciniphila did not mimic prebiotics food intake behaviors. There is also a great interest in $A$. muciniphila because of its association with animal and human health. Remarkably, decreased levels of $A$. muciniphila have been observed in patients with inflammatory bowel disease (mainly ulcerative colitis) and metabolic disorders, suggesting that they may have potential anti-inflammatory properties.[ $[\mathrm{rr}, \mathrm{d}]$

\section{Conclusion}

Several studies have shown that $A$. muciniphila can reduce the serum level of inflammatory cytokines like IL-2, IFN- $\gamma$, IL-12p4O, and MCP-1, and also the lipid overload process linked with the LDL receptor pathway by decreasing apoB48 and apoB10o on LDLs. Meanwhile, A. muciniphila can modulate the immune system by production of short-chain fatty acid which is elaborate in signaling to the host by inhibiting histone deacetylase (HDAC) or by activating G-protein-coupled receptors, which triggers other metabolic pathways [34,36]. The main steps towards the growth of $A$. muciniphila as a new probiotic candidate have been completed. Initially, the identification of $A$. muciniphila being grown on a defined medium according to performance in humans. Second, it has been found that inactivation of the bacteria by pasteurization improves its stability and durability. Third, Amuc_110o introduced as a key mechanism of interaction between $A$. muciniphila and its host. Fourth, the evidence that $A$. muciniphila may be safely managed in the targeted people [9, 17, 35]. Eventually, the pasteurized bacteria and the isolation of bacterial components like the fairly small $30-\mathrm{kDa}$ Amuc_1100 cause to putative progress of drugs based on A. muciniphila related products that could target inflammatory bowel disease or diseases that compromise the function of the bowel barriers [33]. As 
a result, comprehensive information on $A$. muciniphila has become progressively available, and many of the criteria set for evaluating novel food safety in Europe can be fulfilled. However, no studies on the toxicological properties of $A$. muciniphila, including dose-response studies, long-term studies, and reproduction, have been published so far. Such studies are likely to be important before authorities complete a comprehensive safety assessment.

\section{Author Contributions}

All authors contributed equally to this manuscript and approved the final version of manuscripts .

\section{Conflict of Interests}

The authors declare that they have no conflicts of interest.

\section{Ethical declarations \\ Not applicable . \\ Financial Support \\ Self-funding.}

\section{References}

1. Euzéby JP. List of Bacterial Names with Standing in Nomenclature: a folder available on the Internet. Int J Syst Evol Microbiol. 1997;47(2):590-2.

2. Dao MC, Everard A, Aron-Wisnewsky J, Sokolovska N, Prifti E, Verger EO, et al. Akkermansia muciniphila and improved metabolic health during a dietary intervention in obesity: relationship with gut microbiome richness and ecology. Gut. 2016;65(3):426-36.

3. Gómez-Gallego C, Pohl S, Salminen S, De Vos W, Kneifel W. Akkermansia muciniphila: a novel functional microbe with probiotic properties. Benef Microbes. 2016;7(4):571-84.

4. Zhou K. Strategies to promote abundance of Akkermansia muciniphila, an emerging probiotics in the gut, evidence from dietary intervention studies. J Funct Foods. 2017;33:194-201.

5. Everard A, Belzer C, Geurts L, Ouwerkerk JP, Druart C, Bindels LB, et al. Cross-talk between Akkermansia muciniphila and intestinal epithelium controls diet-induced obesity. Proc Natl Acad Sci. 2013;110(22):9066-71.

6. Depommier C, Everard A, Druart C, Plovier H, Van Hul M, Vieira-Silva S, et al. Supplementation with Akkermansia muciniphila in overweight and obese human volunteers: a proofof-concept exploratory study. Nat Med. 2019;25(7):1096-103.

7. Plovier H, Everard A, Druart C, Depommier C, Van Hul M, Geurts L, et al. A purified membrane protein from Akkermansia muciniphila or the pasteurized bacterium improves metabolism in obese and diabetic mice. Nat Med. 2017;23(1):107-13.

8. Belzer C, De Vos WM, Cani PD. Use of a polypeptide for effecting immune signalling and/or affecting intestinal barrier function and/or modulating metabolic status. Google Patents; 2018.

9. Naito Y, Uchiyama K, Takagi T. A next-generation beneficial microbe: Akkermansia muciniphila. J Clin Biochem Nutr. 2018;63(1):33-5.
10. Roubalová R, Procházková $\mathrm{P}$, Papežová $\mathrm{H}$, Smitka K, Bilej M, Tlaskalová-Hogenová H. Anorexia nervosa: Gut microbiotaimmune-brain interactions. Clinical Nutrition. 2020;39(3):67684.

11. Seebeck T, Chen F, Davis GD. Engineered cas9 systems for eukaryotic genome modification. Google Patents; 2019.

12. Cheng P, Yao L, Chen X, Su X, Su X, Huang Q, et al. An inflammatory memory and angiogenic self-assembling nanofiber hydrogel scaffold seeded with Akkermansia muciniphila to accelerate the healing of diabetic ischemic ulcers. RSC Adv. 2018;8(31):17357-64.

13. Remely M, Hippe B, Zanner J, Aumueller E, Brath H, G Haslberger A. Gut microbiota of obese, type 2 diabetic individuals is enriched in Faecalibacterium prausnitzii, Akkermansia muciniphila and Peptostreptococcus anaerobius after weight loss. Endocr Metab Immune Disord Drug Targets. 2016;16(2):99-106. 14. Cani PD, de Vos WM. Next-generation beneficial microbes: the case of Akkermansia muciniphila. Front Microbiol. 2017;8:1765.

15. Gibson GR, Roberfroid MB. Dietary modulation of the human colonic microbiota: introducing the concept of prebiotics. J Nutr. 1995;125(6):1401-12.

16. Roberfroid M. Prebiotics and synbiotics: concepts and nutritional properties. Br J Nutr. 1998;80(S2):S197-S2O2.

17. Rostami FM, Mousavi H, Mousavi MRN, Shahsafi M. Efficacy of probiotics in prevention and treatment of infectious diseases. Clin Microbiol Newsl. 2018;40(12):97-103.

18. Cani PD, Amar J, Iglesias MA, Poggi M, Knauf C, Bastelica D, et al. Metabolic endotoxemia initiates obesity and insulin resistance. Diabetes. 2007;56(7):1761-72.

19. Derrien M, Vaughan EE, Plugge CM, de Vos WM. Akkermansia muciniphila gen. nov., sp. nov., a human intestinal mucindegrading bacterium. Int J Syst Evol Microbiol. 2004;54(5):146976.

20. Ouwehand AC, Derrien M, de Vos W, Tiihonen K, Rautonen N. Prebiotics and other microbial substrates for gut functionality. Curr Opin Biotechnol. 2005;16(2):212-7.

21. Derrien M, Collado MC, Ben-Amor K, Salminen S, de Vos WM. The Mucin degrader Akkermansia muciniphila is an abundant resident of the human intestinal tract. Appl Environ Microbiol. 2008;74(5):1646-8.

22. Everard A, Lazarevic V, Derrien M, Girard M, Muccioli GG, Neyrinck AM, et al. Responses of gut microbiota and glucose and lipid metabolism to prebiotics in genetic obese and diet-induced leptin-resistant mice. Diabetes. 2011;60(11):2775-86.

23. Everard A, Lazarevic V, Gaïa N, Johansson M, Ståhlman M, Backhed F, et al. Microbiome of prebiotic-treated mice reveals novel targets involved in host response during obesity. ISME J. 2014;8(10):2116-30.

24. Yan M, Song M-M, Bai R-X, Cheng S, Yan W-M. Effect of Rouxen-Y gastric bypass surgery on intestinal Akkermansia muciniphila. World J Gastrointest Surg. 2016;8(4):301.

25. Cani PD. Microbiota and metabolites in metabolic diseases. Nat Rev Endocrinol. 2019;15(2):69-70.

26. Macchione I, Lopetuso L, Ianiro G, Napoli M, Gibiino G, Rizzatti G, et al. Akkermansia muciniphila: key player in metabolic and gastrointestinal disorders. Eur Rev Med Pharmacol Sci. 2019;23(18):8075-83.

27. Zhang L, Carmody RN, Kalariya HM, Duran RM, Moskal K, Poulev A, et al. Grape proanthocyanidin-induced intestinal bloom 


\section{Mohammadzadeh Rostami et al.}

of Akkermansia muciniphila is dependent on its baseline abundance and precedes activation of host genes related to metabolic health. J Nutr Biochem. 2018;56:142-51.

28. Anhê FF, Pilon G, Roy D, Desjardins Y, Levy E, Marette A. Triggering Akkermansia with dietary polyphenols: A new weapon to combat the metabolic syndrome? Gut Microbes. 2016;7(2):14653.

29. Cani PD, Osto M, Geurts L, Everard A. Involvement of gut microbiota in the development of low-grade inflammation and type 2 diabetes associated with obesity. Gut Microbes. 2012;3(4):279-88.

30. Ganesh BP, Klopfleisch R, Loh G, Blaut M. Commensal Akkermansia muciniphila exacerbates gut inflammation in Salmonella Typhimurium-infected gnotobiotic mice. PloS One. 2013;8(9):e74963.

31. Hutkins RW, Krumbeck JA, Bindels LB, Cani PD, Fahey Jr G, Goh YJ, et al. Prebiotics: why definitions matter. Curr Opin Biotechnol. 2016;37:1-7.

32. Kaplan M, Mutlu EA, Benson M, Fields JZ, Banan A, Keshavarzian A. Use of herbal preparations in the treatment of oxidant-mediated inflammatory disorders. Complement Ther Med. 2007;15(3):207-16.

33. Jonsson AL, Bäckhed F. Role of gut microbiota in atherosclerosis. Nat Rev Cardiol. 2017; 14(2):79-87.

34. Gülden E, Wong FS, Wen L. The gut microbiota and Type 1 Diabetes. Clin Immunol. 2015; 159(2):143-53.

35. Schneeberger M, Everard A, Gómez-Valadés AG, Matamoros S, Ramírez S, Delzenne NM, et al. Akkermansia muciniphila inversely correlates with the onset of inflammation, altered adipose tissue metabolism and metabolic disorders during obesity in mice. Sci Rep. 2015;5:16643.

36. Xu Y, Wang N, Tan HY, Li S, Zhang C, Feng Y. Function of Akkermansia muciniphila in Obesity: Interactions With Lipid Metabolism, Immune Response and Gut Systems. Front Microbiol. 2020; 11:219. 